\title{
Modelo de operación de la Biblioteca Nacional de Ciencia y Tecnología del Instituto Politécnico Nacional ${ }^{1}$
}

OPERATION MODEL OF THE National Library of Science and Technology of the National Politecnic

Institute

\section{Noel Angulo Marcial* E-mail nangulo@redipn.ipn.mx}

\begin{abstract}
Resumen: Propuesta para un modelo de operación de la Biblioteca Nacional de Ciencia y Tecnología del Instituto Politécnico Nacional, en la que se hacen algunas consideraciones estratégicas con relación a la creación de servicios bibliotecarios, destinadas a la explotación intensiva de los recursos de la información mediante la incorporación de la tecnología y la transición gradual de la biblioteca tradicional a la automatizada y hacia la biblioteca electrónica y su articulación con las funciones sustantivas de la educación superior.
\end{abstract}

Palabras clave: Bibliotecas académicas; Bibliotecas universitarias; Servicios de información; Automatización de bibliotecas; Desarrollo bibliotecario; Planeación de bibliotecas; Biblioteca Nacional de Ciencia y Tecnología; Mexico

\section{Introducción}

La sociedad actual, en la medida que se vuelve más compleja, sustenta cada vez más su desarrollo en la creación, almacenamiento, organización, diseminación y explotación del conocimiento. La creciente producción de literatura científica y técnica, en diversos formatos y soportes, desde el papel hasta los registros legibles por computadora, representa posibles ventajas para quienes tienen la capacidad de recuperar, en forma selectiva y oportuna, aquella información que responde a sus necesidades y propósitos de aplicación. Solesbury (1994) ha dicho que "por definición, el conocimiento debe ser conocido; para que éste pueda ser conocido por alguien más, aparte de su creador, debe ser compartido, y para asegurar la mayor ventaja, su transferencia es tan importante como su creación"

Se habla con frecuencia del derecho a la información como una justa aspiración del hombre, no obstante, el acceso real a ésta sólo se logra en la medida en que se dispone de las habilidades informativas además de los mecanismos formalmente establecidos para el libre flujo de conocimientos entre los distintos sectores de la sociedad; la biblioteca, en este contexto, ha jugado un papel importante al adquirir, organizar y poner a disposición de los interesados, la producción intelectual y los valores de la cultura universal, sin embargo el incremento en el volumen de los registros del conocimiento y su diversificación, hace prácticamente imposible que una sola biblioteca, con el esquema tradicional de operación, pueda cubrir por lo menos los avances que se generan en un campo o disciplina.

Con la proliferación de las redes de computadoras, enlazadas por vía satelital, microondas, fibra óptica y otros medios, se puede ahora disponer de grandes volúmenes de datos, susceptibles de consulta desde la biblioteca y aun en el propio domicilio del usuario; sin embargo algunos de los recursos de mayor rigor académico sólo son disponibles mediante suscripción previo pago de regalías, tal es el caso de 
las bases de datos en línea que sustituyen a las publicaciones periódicas convencionales.

Por otra parte, se aprecia una transición paulatina de las publicaciones científicas hacia la conversión en formato totalmente electrónico y su incorporación en servidores enlazados por redes de alcance mundial; ahora es posible el acceso a una diversidad de recursos, distintos a los medios tradicionales; los cuales se encuentran en diferentes idiomas, condición que continua siendo una barrera para su explotación. Se aprecia, también una diversificación e incremento en la producción de publicaciones y bases de datos en disco compacto y se prevé una mayor aplicación de este tipo de medios pues se dispone de nuevos formatos que incrementan la capacidad de almacenamiento de datos, del orden de 10 gigabytes.

Cabe señalar que las bases de datos en disco compacto resultan excesivamente costosas, por lo cual se precisa establecer rigurosos criterios de selección y diseñar esquemas de operación que permitan extender los beneficios a las distintos sectores de la comunidad de usuarios sin grabar innecesariamente el presupuesto de las bibliotecas, descartando los costos derivados de la duplicidad en suscripciones, mediante la contratación de licencias corporativas para su operación en red.

Ahora resulta más evidente la dificultad para que una persona, por sus propios medios, pueda resolver el problema de acceso pertinente, oportuno, de calidad y confiable a los recursos de información disponibles a nivel mundial y la necesidad de repensar y reestructurar los servicios bibliotecarios y de información para incrementar las posibilidades de apoyo a la comunidad académica y a la sociedad en su conjunto.

Para Blaise Cronin (1992), los servicios de información tradicional han reducido su tarea a una serie de funciones que privilegian el tratamiento documental, que van desde la adquisición hasta la recuperación de materiales. La realización de estas rutinas ha significado, en la mayoría de los casos, el desplazamiento de su función principal: estar al servicio de la estrategia informativa de toda la organización en su conjunto.

\section{Desarrollo Bibliotecario}

Las bibliotecas son entidades dinámicas que requieren, necesariamente, para poder subsistir y cumplir su misión, de una actualización y adecuación permanente a fin de dar respuesta a las necesidades de información de su comunidad. El desarrollo de los servicios bibliotecarios en el Instituto requiere de un cambio cualitativo en la capacidad real y potencial de sus bibliotecas para proporcionar más y mejores servicios. Desde luego, también se necesita prever y planificar la actualización de sus colecciones, la ampliación y readecuación de la planta física, pero fundamentalmente se precisa de la capacitación del personal para asumir un nuevo rol; apoyado en la aplicación creativa y eficiente de la tecnología disponible.

En el caso particular del Instituto Politécnico Nacional, la creación de la Biblioteca Nacional de Ciencia y Tecnología, además de prever la atención a los aspectos tradicionales de toda biblioteca, enfrenta nuevos retos para mejorar la calidad, el impacto y la cobertura de los servicios de información, entre los cuales destacan:

- Alfabetización informacional. Consiste en el desarrollo de las habilidades intelectuales, actitudes y destrezas de su comunidad de 
usuarios para posibilitar la explotación racional de los recursos de la información

- Disponibilidad de estructuras mÁs eficaces. Nuevas formas de organización de la información con el propósito de simplificar el acceso selectivo al conocimiento registrado, como son los catálogos en línea e hipertextos

- ANÁlisis. Contribuir a la explotación intensiva de los recursos de información haciendo explícitas las ideas, mostrando su estructura y relaciones internas para facilitar su comprensión y apropiación

- AdECUACIÓN DE los RECURSOS DE LA INFORMACIÓN. Rediseño de contenidos atendiendo a las características y necesidades específicas de sectores de la comunidad de usuarios

- CONCERTACIÓn DE VOlUntAdes y ACUERDos. Complementación y uso compartido de la información al interior del propio Instituto y en el plano interinstitucional para la optimación de los recursos disponibles

- CONSOlIDACIÓN DE LA INFRAESTRUCTURA DE INFORMACIÓN. Posibilitar el acceso remoto (consulta a distancia), la reproducción y la transferencia electrónica de documentos sin detrimento de su calidad y oportunidad

- $\quad$ TRANSICIÓN DE LA BIBLIOTECA. Prever la evolución gradual y consistente de la biblioteca tradicional a la automatizada y a la biblioteca electrónica

- RACIONALIZACIÓN DEL ACERVO BIBLIOGRÁFICO. Diseñar y operar un modelo para la selección y el desarrollo de colecciones; complementar y optimar la adquisición en propiedad con el acceso a los servicios de información en línea

- DEFINICIÓN DE LA NORMATIVIDAD QUE DARÁ SUSTENTO AL NUEVO ESQUEMA DE OPERACIÓN. Disponer de elementos para regular la operación, adecuación y desarrollo de los servicios bibliotecarios y de información del Instituto

- DEFINICIÓN DE CRITERIOS E INDICADORES. Contar con elementos para la evaluación de los servicios bibliotecarios y de información, buscando un equilibrio entre cantidad y calidad y su articulación con las funciones sustantivas del Instituto

- PROFESIONALIZACIÓN DE LOS SERVICIOS BIBLIOTECARIOS Y DE INFORMACIÓN mediante la incorporación de personal especializado, la capacitación del personal en activo y la operación de un sistema de reconocimiento y certificación de competencias

- $\quad$ Planta física AdeCuAda a los nUeVOS SERVICIOS. Definir con visión prospectiva las áreas funcionales y contar con las instalaciones apropiadas para la operación actual y desarrollo futuro de los servicios bibliotecarios y de información en el contexto de las tecnologías disponibles

- CREACIÓN DE LOS COMITÉS DE BIBLIOTECA E INFORMACIÓN. Involucrar a los distintos sectores de la comunidad académica de las escuelas centros y unidades del IPN en la definición de políticas que orienten la operación y desarrollo de los servicios de biblioteca e información de tal forma que incidan positivamente en la vida académica del Instituto

- VINCULACIÓn CON LA COMUNIDAD. Abrir espacios de comunicación con la comunidad académica, con el sector productivo y con la sociedad para orientar el diseño y promoción de productos y servicios de información que se generen en las bibliotecas y centros de información del Instituto 
- $\quad$ Conformar la Red de Bibliotecas del instituto. Definir el esquema de operación y articulación de las bibliotecas y su incorporación en un sistema de red en el que se privilegie el uso compartido y la complementación de sus recursos

\section{Análisis documental}

Es una actividad, propia de toda biblioteca y centro de información, destinada a identificar y describir el continente y el contenido de documentos en forma distinta a la original, con el propósito de garantizar su recuperación selectiva y oportuna, además de posibilitar su intercambio, comunicación y aplicación. No tendría caso alguno disponer de acervos bibliográficos si se careciera de la posibilidad de identificar los que resultan relevantes a un propósito o necesidad específica.

Para que un artículo, una ponencia, un libro o cualquier otro documento de la colección pueda ser recuperable, se hace necesario desarrollar una serie de actividades previas que generalmente pasan desapercibidas para el usuario común, tal es el caso de la catalogación, la clasificación, la indización, la condensación, las cuales incorporan valor agregado a los recursos disponibles. Mediante la catalogación se describen los rasgos característicos de cada uno de los documentos, la clasificación se destina a asignar una dirección relativa y un orden de precedencia de acuerdo con el tema principal al que se orienta su contenido; la indización aporta, en forma detallada y explícita, los diferentes conceptos que se abordan en el documento, los cuales constituirán los diferentes puntos de acceso. La condensación proporciona una versión abreviada de su contenido.

Si bien la computadora se ha convertido en una herramienta de primer orden en el tratamiento de los recursos de la información, su mayor significación y valor instrumental se obtiene cuando se combina con la aplicación de técnicas documentales y el uso de normas descriptivas de aceptación internacional que dan compatibilidad y solidez a los servicios de información y aportan las condiciones para la cooperación interbibliotecaria en la creación y operación de Catálogos Colectivos de Acceso Público en Línea y las Bases de Datos Bibliográficas. Los catálogos y las bases de datos bibliográficas, consisten de una serie de datos afines en su contenido y cuyas diferentes ocurrencias se estructuran sistemáticamente para controlar la redundancia, mostrar sus relaciones implícitas y facilitar su identificación y recuperación selectiva.

Un sistema de recuperación de información es capaz de manejar las relaciones existentes entre los diferentes registros y permite establecer nuevas relaciones con el propósito de identificar el documento o conjunto de documentos que resultan pertinentes a una necesidad específica de información. El usuario del sistema expresa su necesidad de información mediante un lenguaje de interrogación, el cual puede ser un tesauro o lista de encabezamientos de materia. El sistema de recuperación de información se encarga de evaluar, en forma automática, el nivel de correspondencia entre el contenido de los documentos con respecto a las necesidades de información expresadas por el usuario, para ello se vale de la comparación entre los términos presentes en el perfil de búsqueda y los asignados a cada documento durante el proceso de análisis.

La calidad del análisis documental será de importancia crucial en la generación del Catálogo Colectivo de las Bibliotecas del Instituto y en el desarrollo de bases de datos 
de la literatura científica y tecnológica. Al respecto se prevé la disponibilidad, dentro de la estructura orgánica de la BNCT, de una entidad, dotada del personal y los recursos técnicos, para organizar en forma centralizada los recursos de información que se incorporen en el sistema de bibliotecas del Instituto. ésta tendrá la responsabilidad de unificar los procedimientos de acuerdo a las normas y criterios de uso internacional.

\section{Servicios}

Los servicios bibliotecarios se conforman por el conjunto de apoyos que ofrece la biblioteca, a partir de sus recursos locales, para satisfacer las necesidades de una comunidad; en tanto que por servicios de información se hace referencia a aquellas actividades específicas destinadas a proveer el acceso real a los registros del conocimiento, disponibles en cualquier idioma, tipo de formato, o procedencia, independientemente de que se encuentren en la biblioteca o fuera de ésta. La operación de estos servicios frecuentemente implica la interacción entre el usuario y el proveedor.

Los servicios de información responden a necesidades específicas tales como:

- Realizar búsquedas exhaustivas o de alto nivel de especificidad

- Conocer lo que se ha publicado sobre un tema

- Saber quiénes están publicando en un campo o tema específico

- Conocer lo que ha publicado un investigador

- Conocer los más recientes hallazgos en un campo o disciplina (Estado del arte)

- Acceso al texto completo de una norma, patente, artículo, etc.

La operación de los servicios de información implica el desarrollo de actividades de carácter intelectual destinadas a generar nuevos productos a partir de los documentos primarios para posibilitar su explotación. Implican también, la transformación o descripción analítica de los documentos originales, tal es el caso de la elaboración de índices, resúmenes, bases de datos bibliográficas o de texto completo, de apoyo a la investigación.

La función primordial de una biblioteca es, precisamente, relacionar los recursos de información existentes con las necesidades de su comunidad. Al respecto, la Biblioteca Nacional de Ciencia y Tecnología y la Red de Bibliotecas del Instituto atenderán la prestación de los servicios bibliotecarios tradicionales al mismo tiempo que se iniciará un programa para la transición hacia la biblioteca electrónica; para este propósito se habrá de propiciar una mayor atención a los servicios de información y documentación mediante el uso intensivo de la tecnología, tales servicios se fortalecerán y diversificaran con un nuevo esquema de productos y servicios, a fin de incidir en forma positiva en la calidad de las funciones sustantivas del Instituto.

Uno de los servicios que se habrán de reorganizar para obtener el máximo beneficio de los recursos disponibles es el referente a la diseminación de información, al mismo tiempo que se implantará, en forma automatizada, el Catálogo Colectivo de Acceso al Público y se crearán los servicios de Alerta Bibliográfica, ambos destinados a lograr el uso compartido de los recursos biblio-hemerográficos de las diferentes bibliotecas del Instituto y que se complementarán con un sistema de transferencia de documentos a través de la Red académica del IPN, es decir, la Biblioteca Nacional tendrá la capacidad de proporcionar en forma totalmente electrónica los documentos disponibles en su colección. 


\section{Caracterización de los servicios.}

\subsection{Servicios bibliotecarios}

- $\quad$ PRÉStAmo inTERNO: suministro de todo tipo de material documental para su consulta en los espacios asignados por la biblioteca dentro de sus instalaciones

- $\quad$ PRÉStAMO EXTERNO: suministro de material documental para uso fuera de las instalaciones de la biblioteca, durante un período específico, previa aprobación y registro formal

- $\quad$ PRÉSTAMO INTERBIBLIOTECARIO: obtención de material documental en otras bibliotecas mediante acuerdos de cooperación de beneficio mutuo para las instituciones participantes

- Reserva: disponibilidad de material que se considera complemento indispensable a los programas de estudio, con ello se garantiza la existencia de un número predeterminado de ejemplares para consulta en el interior de la biblioteca, el cual sólo se prestará a domicilio durante los fines de semana

- CONSULTA: asesoría, orientación y asistencia personalizada a los usuarios en el conocimiento, operación y explotación de los servicios y recursos de la información: este servicio se complementa con una colección de referencia, en la que se incluyen diccionarios especializados, enciclopedias, glosarios, boletín de resúmenes analíticos, índices, etc.

- Reprografía: servicio destinado a posibilitar la reproducción de documentos, de todo tipo, para facilitar su consulta, ajustándose a lo estipulado en los derechos de autoría

- PUBLICACIONES PERIÓDICAS: acceso sistemático a la colección de revistas especializadas, disponibles en forma de publicaciones convencionales, en disco compacto, en línea o en una combinación de éstos

- MAPOTECA: disponibilidad de una colección sistematizada de material cartográfico, considerando atlas, mapas y objetos tridimensionales

- $\quad$ ARCHIVO HISTÓRICO: registro sistemático y custodia del material publicado en el Instituto así como la conservación del acervo documental histórico del Instituto, considerando iconografía y archivos para su consulta y explotación sistemática

- Recursos para el APRENDIZAJE: todo tipo de material audiovisual, tridimensional y multimedia susceptible de consulta en la sala de servicio, en la sala de proyecciones de la Biblioteca, o fuera de ésta en las salas de proyección de las distintas escuelas, centros y unidades de enseñanza e investigación

\subsection{Servicios de información}

La consulta a los servicios de información se apoyará en aplicaciones hipertexto (Webs), de tal manera que el usuario podrá interactuar en un ambiente gráfico, seleccionar los recursos a utilizar y obtener ayuda e instrucciones, simplemente presionando el ratón; los servicios de información tendrán diferentes modalidades: 
- $\quad$ la consulta a bases de datos bibliográficos en disco compacto o en LíNEA: Aportan un modelo de representación física y conceptual de artículos y publicaciones, incluyendo su caracterización temática, destinada a precisar el contenido y posibilitar su recuperación selectiva

- $\quad$ EL CATÁLOGO PÚBLICO DE ACCESO EN LínEA, constituido en forma similar a las bases de datos bibliográficas, pero destinado a registrar el acervo de las bibliotecas del Instituto

- $\quad$ ACCESO A BASES DE DATOS FACTUALES Y DE TEXTO COMPLETO, considerando normas y patentes (acceso dedicado)

- $\quad$ El ACCESO A SISTEMAS DE INFORMACIÓN PUNTUAL Y TABLAS DE CONTENIDO: aportan los datos descriptivos de cada uno de los artículos de las revistas consideradas en el segmento contratado, permite conocer la literatura reciente, publicada en revistas científicas internacionales, además de posibilitar su recuperación vía fax, mensajería o transferencia electrónica

- DISPONIBILIDAD DE LITERATURA CIENTÍFICA Y TÉCNICA EN TEXTO COMPLETO mediante discos compacto o a través del Servidor de la Biblioteca, tal es el caso de colecciones de revistas las cuales aportan una imagen facsimilar del artículo o su versión en caracteres Ascii; esta última susceptible de edición

- ACCESO A LOS RECURSOS DE INTERNET: El usuario podrá realizar directamente sus consultas y tendrá la posibilidad de hacer respaldos en disco magnético

- AYUdA EN LíNEA; el usuario podrá tener . además de la asesoría personalizada, acceso a instructivos y guías destinadas al aprovechamiento cabal de los productos y servicios que ofrece la biblioteca

Aunque la biblioteca moderna, exige una mayor iniciativa y responsabilidad del usuario en la operación directa de los sistemas de información de texto completo y catálogos de acceso a los diferentes recursos, el volumen, diversidad y riqueza de las respuestas obtenidas en la consulta, tanto en disco compacto como vía Internet, precisan de nuevos servicios que complementen a los ya establecidos, tal es el caso del diseño y reempaquetamiento.

El reempaquetamiento se ha convertido en un proceso necesario en la explotación de los resultados de la consulta en bases de datos de texto completo y documentos de la red. Básicamente se trata de una reelaboración del contenido o rediseño de su estructura, haciendo uso de bases de datos y procesadores de texto, con el propósito de aportar valor agregado a los datos a fin de darles significación, cuando ésta no se hace evidente en su versión original, mejorar su presentación, eliminar redundancias, precisar la fuente y fundamentalmente adecuarlo a las características específicas de diferentes sectores de la comunidad.

Los cambios que ahora se presentan en los servicios de la biblioteca y los centros de información se deben, en buena medida, a la aplicación de la tecnología de la información. Neelameghan, citado por Lancaster (1993), representa esta evolución de los servicios en el siguiente cuadro que se modifica para adecuarlo a la biblioteca:

\begin{tabular}{|c|l|}
\cline { 2 - 2 } Valor & USUARIOS: DECISIONES \\
Asesoría / Consultoría \\
Análisis de tendencias / Pronósticos \\
Estado del Arte. Revisión de la Literatura \\
Análsis, Extracción. Recuperación \\
Síntesis de la Información \\
Diseño y Reempaquetamiento \\
Indización y Condensación. Abstracts \\
Catalogación y Clasificación \\
Selección y Adquisición. Búsqueda
\end{tabular}




\section{Información}

\section{Cuadro 1}

El valor agregado responde a las necesidades específicas de los usuarios. La preparación de productos y servicios de valor agregado requiere de personal profesional especializado, que reúna:

- Un profundo conocimiento del área de especialidad de los usuarios

- Habilidad en el manejo de las herramientas básicas de la computación

- $\quad$ Alto nivel de competencia en información

- Conocimiento de la metodología de construcción y difusión del conocimiento científico

- $\quad$ Capacidad y experiencia para interactuar y trabajar con especialistas del campo y con el usuario final

- Conocimiento de las técnicas y metodologías del análisis, consolidación y reempaquetamiento de la información

\subsection{Servicios de extensión}

- $\quad$ Conferencias. Teleconferencias

- $\quad$ Exposiciones

- $\quad$ Conciertos

- Ludoteca

- $\quad$ Proyección de películas, videos

- Intercambio bibliotecario

- Sala de cursos

- $\quad$ Desarrollo de habilidades informativas (Educación de usuarios)

\subsection{Servicios adicionales}

- $\quad$ Salas de seminarios

- Laboratorio de Tecnología de la Información

- $\quad$ Cubículos de estudio grupal e individual

- $\quad$ Salas de proyección

- $\quad$ Cubículos de computadoras, material audiovisual, etc.

- Vestíbulo / Área de exposiciones

- $\quad$ Centro de Apoyo a Estudiantes (CAE)

- Centro de capacitación para la operación de fuentes de información en línea

- Librería

\section{Calidad de los servicios}


Mejorar la calidad y pertinencia de los servicios para propiciar el uso intensivo de los recursos disponibles significa también crear un ambiente digno para la interacción entre los usuarios, los registros del conocimiento y los medios de acceso a la información, ampliar los espacios de atención, mejorar las condiciones de mobiliario, equipo e instalaciones, y fundamentalmente, diversificar e incrementar las posibilidades de acceso a los registros del conocimiento científico y tecnológico, contratando servicios de información puntual, abriendo convenios de colaboración con instituciones de educación superior e investigación y generando en su caso, aplicaciones propias que aseguren la oportunidad en el tiempo de respuesta y la obtención de economías mediante la complementación de acervos, la adquisición y uso compartido de los recursos disponibles localmente, evitando la duplicidad innecesaria de colecciones y posibilitando una mayor actualización y cobertura temática.

Es frecuente que la evaluación de la calidad de los servicios se sustente en la comparación con normas bibliotecarias, sin embargo se pierde de vista la apreciación del usuario, quien es la razón de ser de la biblioteca. Francoise Hébert (1995) advierte que "la decisión de usar los servicios de la biblioteca y de volver a utilizarlos, o recomendarlos a terceros, depende de una evaluación subjetiva de la interacción con los servicios y no de la correspondencia de éstos con una norma institucional".

Por otra parte, la definición de parámetros de orden cuantitativo ${ }^{2}$ ha sido de enorme utilidad en el pasado cercano para garantizar la disponibilidad de recursos documentales en las bibliotecas, y para establecer condiciones mínimas de acceso a la colección y a los servicios. Lo anterior explica la tendencia, aún vigente en las bibliotecas más conservadoras, de fijar criterios cuantitativos, que la mayor de las veces no se cumplen, para valorar el desempeño o la calidad de los servicios, tal es el caso del número publicaciones por usuario potencial; número de revistas por área de conocimiento, especialidad o estudios de posgrado; número de títulos por alumno, etc.

La aplicación de indicadores cuantitativos ha venido orientando el desarrollo de bibliotecas que ahora se enfrentan al problema del incremento en el costo de las publicaciones periódicas, la insuficiencia presupuestal para cumplir con los parámetros hetero-proyectados y la carencia del espacio requerido para almacenar el volumen de publicaciones que se considera necesario para apoyar los programas académicos y de investigación. Al respecto la BNCT, no puede ignorar las normas y parámetros que tradicionalmente han servido de referencia en las bibliotecas, sin embargo deberán generarse nuevos criterios y normas que permitan equilibrar calidad y cantidad.

\section{Distribución de productos y servicios}

La logística se ocupa de las técnicas y procedimientos para hacer llegar recursos o servicios en donde éstos se necesitan o donde serán utilizados; implica llegar al lugar adecuado, en el tiempo adecuado y a un costo adecuado. Las bibliotecas, pese a ser organismos de servicio social, frecuentemente se olvidan de identificar al segmento de usuarios a los que deberían atender, la logística en la prestación de servicios de información parece no tener mayor importancia para los prestadores de este servicio en la biblioteca tradicional, lo cual se explica por sus limitaciones ancestrales, que ahora es posible corregir mediante el uso de la tecnología de la información.

Cómo y dónde se prestan los servicios de información, y de que mecanismos se dispone para hacer llegar la información a los usuarios debe ser una preocupación constante de la biblioteca. Cuando la información no es entregada en el marco de 
tiempo requerido, se reduce su valor de utilidad; si esta no se entrega en el lugar adecuado y en forma oportuna se corre el riesgo de que pierda todo valor de utilidad. Si se proporciona en cantidad insuficiente, no se cumplirán las expectativas del usuario, por el contrario un exceso en la respuesta sobrecargará al usuario con datos que difícilmente podrá utilizar. Si se carece de control sobre la calidad de los productos y servicios de información el usuario tendrá una imagen negativa de los servicios y si no existe facilidad de acceso a los servicios de información el usuario tenderá a prescindir de éstos (Spafford) .

El usuario estará dispuesto a dedicar tiempo, esfuerzo y aún dinero si el producto o servicio es de calidad superior $\mathrm{y}$, desde luego, siempre que responda a sus necesidades e intereses.

Dado que la biblioteca es un medio para apoyar las funciones sustantivas de su Institución, la generación y distribución de productos y servicios de información debe considerar la segmentación de su comunidad, esto implica la clasificación de usuarios en grupos de acuerdo a su ubicación, afinidad, actividad principal y nivel académico para planear en forma eficaz la atención a cada uno de los segmentos identificados (Montenegro 1994). En cada caso será necesario hacer los siguientes cuestionamientos:

- ¿Los productos y servicios de información responden a los intereses y necesidades del segmento de usuarios a los que se destinan?

- ¿Es necesario desarrollar nuevos productos y servicios para dar respuesta a un nuevo segmento de usuarios?

- ¿Cómo lograr que la comunidad demande y haga uso de los servicios bibliotecarios y de información?

- ¿Es posible mejorar y hacer atractivos los productos de información generados en la biblioteca?

- ¿Las guías e instructivos de los servicios y productos son claras y sencillas de consultar?

- ¿La obtención de documentos es oportuna?

\section{Productos}

La Biblioteca Nacional de Ciencia y Tecnología tendrá la capacidad de generar productos documentales diversos, desde la elaboración y compilación de bibliografías, boletines de alerta, boletines bibliográficos, tablas de contenido, hasta productos de alto valor agregado, como se anota:

- DISEMINACIÓN SELECTIVA DE LA INFORMACIÓN: consiste en la selección y entrega periódica de referencias biblio-hemerográficas con valor de actualidad, conforme al perfil del usuario. Este servicio se destina a investigadores y personal académico, previa autorización y definición del perfil

- Diseño y REEMPAQUetAmiento: supone un papel activo de la biblioteca mediante la adecuación y adaptación de contenidos a las necesidades de los distintos sectores de la comunidad de usuarios. Implica actividades de análisis, re-escritura, traducción y diseño de formatos de presentación 
- $\quad$ BOLETín ELECTRÓNICO: servicio disponible en Internet destinado a propiciar y posibilitar la participación del usuario en grupos de discusión, de tal manera que puede incorporarse en un foro de debates, hacer preguntas, 0 enviar un mensaje simultáneamente a varios suscriptores

- TRAducción de aRTículos CIENTíficos: posibilidad de contar con una versión al español de los artículos de mayor actualidad en el campo de interés del usuario

\section{Componentes del proyecto de creación de la BNCyT}

\subsection{Recursos Humanos}

Sin duda alguna el personal tiene un papel determinante en la proyección de los servicios bibliotecarios y de información; en ese sentido deberá fortalecerse la capacidad y conocimientos del personal de la biblioteca para que en forma individual se constituyan en agentes promotores, facilitando el flujo de la información entre la comunidad de usuarios, interesándolos e induciéndolos al uso intensivo de la información. En la concepción del proyecto de la Biblioteca Nacional de Ciencia y Tecnología no se han soslayado aspectos fundamentales que contribuyen a la obtención de niveles de excelencia, tal es el caso de:

- DEFINICIÓN DE NORMAS DE COMPETENCIAS que constituyan el referente obligado para la incorporación, capacitación y promoción del personal de las bibliotecas del Instituto, considerando la idoneidad con las funciones y objetivos de la biblioteca y la calidad de los servicios

- LA CAPACITACIÓN Y ACtUALIZACIÓN DE lOS RECURSOS hUMANOS ya incorporados en las bibliotecas y centros de información del Instituto, la cual tiene una correspondencia directa con los alcances y niveles de calidad de los servicios

- $\quad$ El RECONOCIMIENTO FORMAL DE LA EXPERIENCIA ADQUIRIDA EN EL EJERCICIO LABORAL, la cual se incorporará en un programa de certificación de competencias que contribuya a consolidar el interés, la identificación y el compromiso del personal con los objetivos institucionales

- LA INCORPORACIÓN DE PERSONAL ESPECIALIZAdo EN EL CAMPO DE LA INFORMACIÓN la cual se traduce en niveles más elevados de desempeño y reduce la inversión de tiempo y dinero en la formación de recursos humanos de alto nivel

- $\quad$ LA CREACIÓN DE UN NUEVO ESQUema DE PLAZAS ACADÉmICAS destinadas a incorporar, en su caso, promover y retener al personal profesional con experiencia en el campo de la bibliotecología, la documentación y la información

\subsection{Recursos de la información}

Se plantea una mayor orientación de los servicios de información en la atención de necesidades "justo en el momento", lo cual supone que se cambiará gradualmente la tendencia a acumular recursos "por si acaso se requieren", hacia la operación de servicios de información puntual, publicaciones electrónicas y bases de datos en línea, 
los cuales se apoyarán en la infraestructura de redes del instituto y en una más abierta relación de intercambio y colaboración con otras instituciones.

Se buscará apoyar con mayor compromiso las tareas de docencia e investigación, mediante el diseño e implantación de un modelo destinado a la selección y desarrollo de colecciones, utilizando criterios académicos, entre otros la conformación de colecciones núcleo en las que estarán representadas, de manera selectiva, las publicaciones periódicas que respondan a las características y necesidades de las distintas disciplinas que se imparten en el Instituto.

El concepto de Colección núcleo significa que se dará atención prioritaria a la adquisición de publicaciones periódicas de las que se espera una mayor demanda, por tener una relación directa con las áreas de conocimiento de importancia estratégica para el Instituto. Las colecciones núcleo estarán disponibles en su forma impresa o en formatos legibles por computadora, en tanto que se tendrá acceso a servicios de alerta y bases de datos en línea que, en forma complementaria, permitirán estar al tanto de los avances en el campo de la ciencia y la tecnología, al mismo tiempo que resuelven el acceso y recuperación de artículos publicados en revistas de todo el mundo.

Por otra parte, la Biblioteca Nacional de Ciencia y Tecnología, tendrá entre sus funciones la colecta y organización de las publicaciones del área científica y tecnológica que se generan en el país, por lo que, además de disponer de recursos propios para la adquisición de acervos bibliográficos, tendrá la posibilidad de enriquecer significativamente sus colecciones de revistas especializadas mediante el intercambio intensivo de las publicaciones periódicas publicadas por el Instituto y la promoción del depósito legal.

\subsection{Tecnología de la información}

La tecnología de la información designa la confluencia de métodos y técnicas, asociadas a la computación y las telecomunicaciones, destinadas a la adquisición, producción, almacenamiento, proceso, transferencia y diseminación de datos contenidos en señales de naturaleza acústica, textual, óptica o electromagnética. La incorporación de la tecnología de la información juega un papel importante en el desarrollo de la sociedad; José Rincón (1994) incluye entre las consecuencias que se derivan de su aplicación las siguientes:

- Mayor integración y acercamiento entre grupos humanos y sociedades organizadas

- Expansión de las áreas de conocimiento y de su aplicación en el quehacer cotidiano

- Mayor tendencia a la automatización en los procesos de adquisición y organización del conocimiento

- Oferta creciente de información en las diversas áreas del conocimiento y campos de la actividad humana

- Necesidad de desarrollo y aplicación de una interfase entre el hombre y los dispositivos de registro destinados al procesamiento del conocimiento científico

- Comunicación precisa, concisa, rápida y cada vez con mayor libertad y cobertura geográfica

Sin duda alguna la tecnología de la información habrá de transformar drásticamente la forma de operación de las bibliotecas y la forma de generación y transferencia de los registros del conocimiento, no obstante para que exista una biblioteca virtual no basta con estar conectado a fuentes de información 0 enlazado a redes de telecomunicación, se precisa que la biblioteca sea organizada y administrada en 
función de las condiciones que impone el acceso a distancia, se requiere de una mayor competencia en información del personal y de los usuarios, además de una sólida infraestructura tecnológica para dar soporte a este modelo de operación lo que implica una cantidad suficiente de equipos para los usuarios y un sistema de puertas entre la red local y las redes externas (Lupovici 1995).

Internet es una fuente de información en red, en constante crecimiento; su desarrollo ha sido azaroso y caótico, pese a que se han hecho algunos esfuerzos para darle una estructura mediante el desarrollo de herramientas de búsqueda y organización de la información. Los recursos disponibles vía Internet pueden tener, diferentes modalidades: archivos de documentos, programas, imágenes; servicios como catálogos de acceso a la biblioteca o directorios o grandes archivos de datos, tal es el caso de resultados de investigaciones o datos experimentales.

La información en red puede estar en forma de textos, datos, imágenes y sonidos, los que. se ponen a disposición de los usuarios de Internet en forma espontánea, sin la mediación de procedimientos sistemáticos de validación o instituciones responsables de valorar y sancionar su calidad, las herramientas de exploración en red aún no alcanzan la consistencia y precisión de las funciones de búsqueda complejas y sofisticadas de los sistemas de información en línea. Lo anterior hace necesaria la mediación de la biblioteca para apoyar en la búsqueda, recuperación y explotación de la información en red y la disponibilidad de programas orientados al desarrollo de las habilidades informativas.

La posibilidad de combinar, en forma totalmente interactiva, texto, imagen, sonido y color abren posibilidades ilimitadas para su aplicación en el desarrollo de nuevos productos y servicios, de los cuales ya se empiezan a difundir algunos avances. Los WEBs y otras formas de hipertexto, combinadas con las telecomunicaciones son también parte de esta nueva realidad, la cual motiva la propuesta de inclusión de la División de Tecnología de la Información en la estructura formal de la biblioteca además de la propuesta de consolidación de la infraestructura de la información del Instituto, considerando el sistema de redes y la automatización de procesos y servicios:

\subsection{Sistema de operación de redes.}

Dadas las características del servicio que se propone, la Biblioteca Nacional de Ciencia y Tecnología debe contar con un servidor para posibilitar la consulta de sus recursos, las 24 horas del día, durante los 365 días del año, sin restricciones geográficas, tanto desde las bibliotecas del Instituto o de las bibliotecas de otras instituciones, así como desde el domicilio de los suscriptores.

Cabe señalar que, cada una de las bibliotecas del Instituto, está o estará dotada con una red local, destinada a la operación y prestación de los servicios, considerando el control de préstamo mediante código de barras, control de adquisiciones, registro de publicaciones periódicas, consulta del catálogo de acceso público en línea, acceso a bancos de información, correo electrónico, etc. A través de Internet, cada biblioteca tendrá la capacidad para conectarse, entre sí y con bibliotecas de otros países.

\subsection{El sistema de automatización integral de bibliotecas}

Debe permitir la operación de bases de datos en redes de área local, la consulta del catálogo público en línea, deberá ser flexible y disponer de una alta capacidad de recuperación y versatilidad para operar búsquedas sencillas y de alto nivel de 
precisión mediante el uso de operadores booleanos y de relación, vía comandos e interfase gráfica, debe posibilitar la operación de subconjuntos de la bases de datos por nodo y en forma colectiva por la agregación de las bases de datos de cada nodo; además deberá disponer de la opción de transferencia de resultados entre los usuarios, todo esto en un ambiente cliente-servidor, mediante el empleo de protocolos $T C P / / P^{3}$

Por otra parte, la automatización deberá liberar al personal de operaciones repetitivas, al mismo tiempo que aporte una mayor confiabilidad y oportunidad en la realización de éstas, tal es el caso del control de préstamos, registro de usuarios, control de acceso, seguimiento presupuestal, control de adquisiciones, composición de los acervos, actualidad de la colección, estadísticas de servicio, registro de publicaciones periódicas, reclamación de adeudos, elaboración de pedidos, elaboración de inventarios, etcétera.

\subsection{Laboratorio de tecnología de la información}

La explotación máxima de los recursos de la información estará sustentada en la disponibilidad de un laboratorio equipado con recursos y personal capacitado en el diseño y desarrollo de aplicaciones y la generación de productos de información, tal es el caso de hipertextos, hipermedios, boletín electrónico, archivos gopher, sitios Web, publicaciones electrónicas, etc.

\subsection{Definición de necesidades}

\subsubsection{Software}

Se requiere realizar un análisis comparativo de los sistemas y aplicaciones disponibles en el Instituto y en el mercado contra los nuevos servicios que se propone incorporar, considerando la compatibilidad con los equipos en existencia y las normas de aplicación en las bibliotecas y centros de información.

\subsubsection{Hardware}

Se prevé un análisis comparativo de la capacidad instalada contra las necesidades detectadas para subsanar las carencias actuales y las necesidades generadas como consecuencia de la implantación del nuevo Proyecto en un horizonte de corto mediano y largo plazo.

\subsubsection{Knoware (recursos de la información)}

Se precisa de la valoración de los recursos disponibles en formatos convencionales, frente a las ventajas de su adquisición en disco óptico, además de la valoración de las sistemas de acceso en línea, tanto bases de datos bibliográficas, como tablas de contenido y publicaciones de texto completo.

\subsubsection{Recursos presupuestales.}

La implantación del modelo de operación implica costos de adquisición, operación, mantenimiento y actualización de cuatro componentes fundamentales requeridos en el proceso de automatización e incorporación de la tecnología de la información: equipo, sistemas, recursos de la información y capacitación del personal, en todos los casos desde la perspectiva actual y su tendencia en el corto, mediano y largo plazo. 


\subsubsection{Estado del arte en tecnología de la información}

La adquisición de equipo y sistemas debe considerar su vigencia y correspondencia con los avances y tendencias en el campo de la tecnología. Decidir con base en el costo no es siempre la mejor alternativa, al respecto se deberá prever la compatibilidad con las normas de uso internacional, el soporte técnico, su complementariedad con la infraestructura instalada, además de someterse a una evaluación rigurosa en cuanto a su factibilidad y ventajas derivadas de su aplicación, y su período de vida útil.

\subsubsection{Diseño e implantación del programa de automatización y aplicación de la tecnología de la información}

El desarrollo o adopción de un sistema de automatización de bibliotecas debe prever su compatibilidad con los sistemas que operan en las principales bibliotecas del mundo y su capacidad de conectividad, además de la incorporación de los avances tecnológicos, siempre que éstos contribuyan a darle una ventaja estratégica que redunde en beneficios tangibles para la comunidad de usuarios, al respecto se precisa de la definición de necesidades y estudio de costos de operación, actualización y mantenimiento.

\section{Objetivos de la BNCyT}

El proyecto de creación de la Biblioteca Nacional de Ciencia y Tecnología se propone alcanzar los siguientes objetivos:

\section{Objetivo general}

- $\quad$ Contribuir al logro cabal de las funciones sustantivas del Instituto, mediante la prestación de servicios de información sustentados en el desarrollo y disponibilidad de colecciones propias y el acceso intensivo a recursos de información internos y externos vía red de computadoras

\section{Objetivos específicos}

- Replantear la estructura ogánico-funcional de los servicios bibliotecarios y de información

- Diseñar y construir el edificio de la Biblioteca Nacional de Ciencia y Tecnología

- Incorporar nuevos servicios destinados a propiciar el uso intensivo de los recursos de la información

- Consolidar la infraestructura de información para la operación de los servicios mediante el uso de nuevas tecnologías

- Diseñar e implantar un programa para la selección y desarrollo de colecciones

- Diseñar e implantar un programa de profesionalización de los servicios de información

- Implantar un programa de formación de usuarios en la cultura de la información 
- Establecer el marco normativo de los servicios bibliotecarios y de información del Instituto

- $\quad$ Establecer acuerdos con las instituciones de educación superior para el uso compartido de los recursos de la información

- Promover la participación de los distintos sectores de la comunidad académica del Instituto en el desarrollo de los servicios bibliotecarios y de información.

\section{Estructura orgánica}

La estructura y modelos tradicionales de operación de los servicios bibliográficos y de información precisan de una adecuación a las nuevas condiciones del entorno; dar el primer paso hacia una nueva concepción de servicios bibliotecarios significa replantear sus alcances y su inserción e impacto en la vida académica, significa también la necesaria evaluación permanente de su desempeño y la aplicación de ajustes.

Los viejos modelos y criterios de operación bibliotecaria, se han venido reproduciendo y aplicando sin una adecuación sistemática a las características y necesidades propias de cada Institución. En la reorganización de las bibliotecas del Instituto, se deben considerar sus características internas, el estado del arte en cuanto a los recursos y la tecnología de la información, la infraestructura disponible localmente, el perfil del personal y los requisitos de formación derivados de los servicios que se habrán de proporcionar y la experiencia adquirida por el personal de sus bibliotecas.

La Biblioteca Nacional de Ciencia y Tecnología no puede ser una entidad pasiva, sino flexible y abierta al cambio, por ello se hace necesario imaginar nuevas formas de articulación con la docencia, la investigación y la difusión. No se propone crear una biblioteca monumental sino el nodo de una red de bibliotecas, responsable de articular los servicios y promover su desarrollo, a fin de brindar condiciones óptimas para contribuir al logro de los objetivos institucionales.

La calidad de nodo central de la red de bibliotecas, la convierten en el eje del cambio en el uso de los recursos de la información y la generación del conocimiento, este compromiso requiere de personal de alto nivel, capaz de incidir en las distintas facetas de operación de las bibliotecas y centros de información del Instituto y participar en el desarrollo de una cultura de la información.

La gran ventaja del Instituto estriba en el hecho reconocido de que parte de una escasa tradición bibliotecaria y aún no existen estructuras consolidadas en la práctica cotidiana, ésta es una posibilidad real de emprender la reorganización de las bibliotecas con una visión futurista, sin depender de un modelo anquilosado e insensible al cambio, aunque también existe el riesgo de caer en la improvisación y la pérdida del rumbo si esta reorganización no se inserta en forma coherente y articulada con la vida académica. La biblioteca no puede verse más como un mero accesorio del que se pueda prescindir sino como uno de los recursos fundamentales para promover el aprendizaje. 


\section{ESTRUCTURA ORGÁNICA}

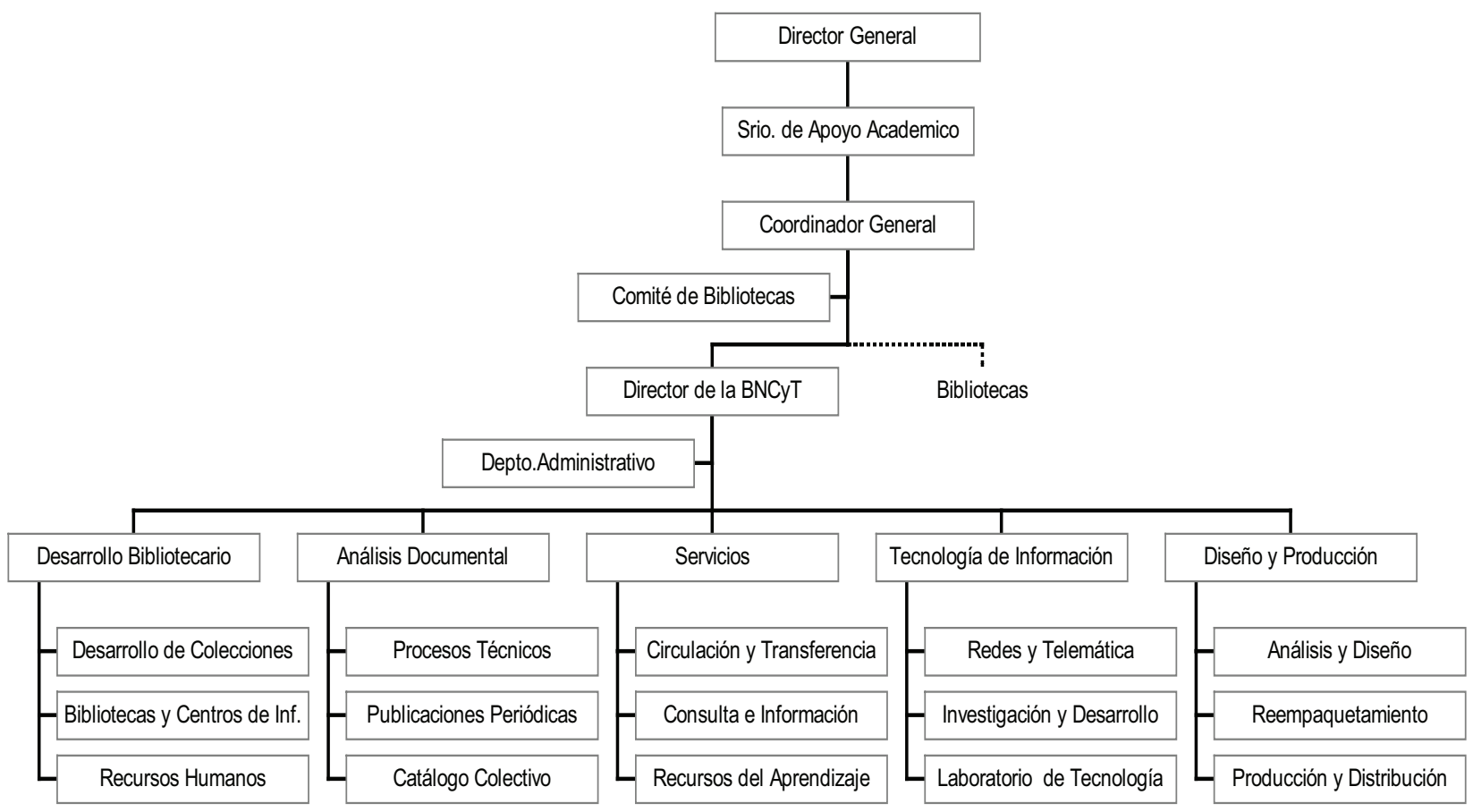

Figura 1

\section{Biblioteca Nacional de Ciencia y Tecnología}

- $\quad$ Primer Nivel:

Director

- $\quad$ Segundo Nivel:

Jefaturas de División

- $\quad$ Tercer Nivel:

Jefaturas de Departamento

I. Desarrollo bibliotecario. Coordinar: el desarrollo y complementación de colecciones, la aplicación de indicadores y normas para mobiliario y equipo de las bibliotecas y servicios de información del Instituto; aplicación de normas para la construcción, mantenimiento y adecuación de la planta física destinada a la operación de bibliotecas y servicios de información; vinculación con las áreas académicas del Instituto y con el sector productivo; concentrar y actualizar la estadística de operación del sistema de bibliotecas; promover las actividades de los comités de biblioteca de cada una de las dependencias del Instituto; concentrar y actualizar los indicadores de evaluación de los servicios de cada una de las bibliotecas y centros de información del Instituto; verificar la idoneidad y pertinencia del personal asignado a los servicios bibliotecarios y de información; identificar y registrar las necesidades de capacitación del personal técnico especializado de las distintas bibliotecas; elaborar el programa de capacitación para el personal de bibliotecas y los servicios de información; Revisar y actualizar las normas de competencia del personal de bibliotecas. 
II. Análisis documental. Coordinar el proceso técnico del material bibliográfico y documental del Instituto; generar y actualizar el catálogo de tesis; desarrollar y actualizar los catálogos de autoridad de materia y autor; desarrollo y adecuación de vocabularios controlados de aplicación en la creación de bases de datos bibliográficas y la generación de registros de los recursos de la información, en sus diferentes modalidades; catalogación y clasificación de las colecciones y generación del catálogo colectivo de acceso al público generar y actualizar el catálogo colectivo de publicaciones periódicas del área tecnológica; desarrollar y actualizar una colección de fuentes documentales auxiliares para las tareas de análisis documental; actualizar la estadística de producción; generar y actualizar el directorio de bibliotecas del área tecnológica

III. Servicios. Coordinar la prestación de servicios de préstamo interno, préstamo externo, préstamo interbibliotecario, transferencia electrónica de documentos, consulta, acceso a bases de datos en disco óptico y en línea; control y acceso sistemático a las publicaciones periódicas; registro de usuarios, acceso a recursos del aprendizaje; mapoteca; fototeca, ludoteca; extensión de los servicios; asignación de cubículos para estudio en grupo; actividades destinadas a promover la lectura y el uso de los recursos de la información; elaborar la guía de acceso a los servicios bibliotecarios y de información

IV. Tecnología de la información. Coordinar la evaluación de sistemas; diseño y desarrollo para dar soporte a los sistemas de información en bibliotecas y centros de información del Instituto; evaluación e incorporación de nuevas tecnologías en el diseño y producción de publicaciones electrónicas; digitalización y codificación de documentos; operación y mantenimiento de los sistemas de información; mantenimiento de la red de bibliotecas: investigación, evaluación y prueba de equipos y programas requeridos en la prestación de servicios; operación del laboratorio de tecnología de la información: elaboración de manuales de operación de sistemas; capacitación en la operación de los sistemas de información a la comunidad académica y a usuarios externos; reproducción de documentos

V. Diseño y producción. Coordinar la transcripción, readecuación, reescritura, traducción, análisis y síntesis de la literatura científica y tecnológica incorporando valor agregado; diseño y elaboración de documentos hipertexto y otros productos de infoirmación; elaboración de la página WEB de la biblioteca; elaboración de estudios de mercado para conocer las necesidades de información del sector productivo; promoción de productos y servicios de información generados en el Instituto; comercialización de los servicios de información para usuarios externos; elaboración del perfil de necesidades de los usuarios; diseño y operación de los servicios de diseminación selectiva de información; edición del órgano informativo de la Biblioteca Nacional de Ciencia y Tecnología.

\section{Implicaciones}

La transición de la biblioteca convencional, a la biblioteca automatizada hasta llegar a la biblioteca electrónica ${ }^{4}$ implica retos y oportunidades, además de una opción para abatir rezagos. El Instituto tiene ahora la oportunidad de obtener un liderazgo en el campo de las bibliotecas técnicas y consolidar un esquema de cooperación interinstitucional que contribuya al cumplimiento cabal de sus funciones sustantivas y 
mejoramiento de la calidad de sus servicios, además de extender sus beneficios a las instituciones de educación tecnológica y al sector productivo.

El diseño de un nuevo esquema de servicios bibliotecarios y de información se sustenta necesariamente en la visión compartida de un nuevo modelo educativo en el que se plantean los siguientes componentes del perfil del egresado:

- Los conocimientos para la clara conceptualización, análisis, diseño, implantación y operación de proyectos

- El desarrollo de habilidades para el diseño y la creatividad tecnológica

- El conocimiento y dominio de la tecnología de la información y las telecomunicaciones

- El desarrollo de habilidades prácticas para la gestión y acción emprendedora, con valores éticos que orienten el desarrollo profesional dentro de una mística de servicio.

La consolidación de la infraestructura de la información, entendida como el conjunto de medios para posibilitar el acceso selectivo al conocimiento científico y la tecnología, considerando colecciones, sistemas, redes y personal capacitado en la explotación de los recursos de la información, contribuirá en forma determinante a los propósitos de dicho modelo. La Biblioteca Nacional de Ciencia y Tecnología, promoverá el desarrollo de las habilidades informativas de su comunidad como una estrategia para lograr el uso intensivo de los recursos disponibles y la explotación racional de las ideas y el conocimiento.

Se advierte que el adjetivo nacional se sustenta en la cobertura de las acciones del Instituto y particularmente en la vocación de la biblioteca para constituirse en el centro de excelencia en materia de información científica y tecnológica en México, no obstante, por el momento no reúne las características propias de las bibliotecas nacionales, lo cual no excluye la posibilidad de evolución hacia esta categoría.

M. en C. Noel Angulo Marcial / Instituto Politécnico Nacional. (México) 1996, revisada en abril de 2001. Comentarios: nangulo@redipn.ipn.mx

\begin{abstract}
Notas
${ }^{1}$ Una versión abreviada de este documento fue publicada en la Revista IPN, Ciencia, Arte: Cultura. Año 3, no. 17, 1998, actualmente se prepara la revisión del modelo y una propuesta de adecuación al contexto de globalización y a un ambiente donde la biblioteca adquiere mayor compromiso con el proceso de aprendizaje y el desarrollo de competencias en información para contribuir al proceso de conversión de la información en conocimiento.

${ }^{2}$ Cabe mencionar que dichos parámetros sólo han representado un elemento parcial de referencia, por ejemplo, en el caso de los acervos bibliográficos, al carecer de políticas y normas para el desarrollo de colecciones, éstas se forman frecuentemente sin correspondencia con criterios de calidad, pertinencia, existencias, costo, autoridad o vigencia, convirtiéndose en una colección obsoleta y de escaso valor instrumental, por otro lado, la interpretación ortodoxa de la norma puede entorpecer la operación de la biblioteca.

3 TCP.IP: Transsmission Control Protocol. Internet Protocol. Protocolos requeridos en el control de transferencia de datos a través de Internet; $I P$, se refiere a las especificaciones para los paquetes de datos. Frecuentemente designan a los programas requeridos en una computadora para conectarse vía Internet.

${ }^{4}$ La biblioteca electrónica es una justa aspiración pero no significa la sustitución drástica del documento impreso como soporte del conocimiento, en el futuro cercano se prevé la coexistencia del soporte impreso, tal como lo conocemos, con diferentes formatos susceptibles de transferencia electrónica y de lectura en pantalla. La biblioteca digital se percibe, en el corto y mediano plazo, como un recurso más de la biblioteca automatizada y no como el remplazo obligado.
\end{abstract}


Summary: Proposal for an operation model of the National Library of Science and Technology of the National Polytechnic Institute, in which are done some strategic considerations in relation to the creation of library services, destined to the intensive use of the information resources by means of technology incorporation and gradual transition of the traditional library to automation and toward the digital library and their articulation with the academic functions of higher education.

Key words: Academic Libraries; University Libraries; Information Services; Library Automation; Library Development; Library Planning; National Library of Science and Technology; Mexico

\section{Referencias}

Cronin, Blaise y Reyes Vila-Velda (1992). Gestión de la inteligencia corporativa: revalorizar los activos informativos de una empresa. (mecanograma).

Hébert, Francoise (1995). La qualite des services: una recherche sur le prét entre bibliothèques dans les grandes bibliothèques publiques du Canada. Documentation et bibliothèques. V. 41, n.4, p.217-224.

Lancaster, F.W., Editor (1993). Libraries and the future: essays on the library in the twenty-first century. New York: The Haworth Press, p. 93.

Lupovici, Christian (1995). Agades: la creación de una biblioteca virtual. Interfase: ciencia y tecnología de Francia, n. 53 (octubre -diciembre).

Montenegro de Lima, Reggina, Célia (1994). Marketing de produtos de informaçao. Ciencia da informaçao, v. 23, n. 3:373-376

Rincón Ferreira, José (1994). O impacto da tecnología da informacão sobre o desenvolvimento nacional. Ciencia da informacão. Brasilia. v. 23, n.1:9-15.

Solesbury, William (1994). Knowledge transfer: the medium and the message. Research evaluation, v. 4 , no. $155: 5$

Spafford, George The logistic of information. http://execpc.com/il/logistic.html

* Instituto Politécnico Nacional, México 